\title{
Relationship between Fetuin A, Vascular Calcification and Fracture Risk in Dialysis Patients
}

\author{
Hung Yuan Chen ${ }^{1,2}$, Yen Ling Chiu ${ }^{1,2}$, Shih Ping Hsu ${ }^{1,2}$, Mei Fen Pai ${ }^{1,2}$, Ju Yeh Yang ${ }^{1,2}$, Yu \\ Sen Peng ${ }^{1,2 *}$
}

1 Division of Nephrology, Department of Internal Medicine, Far Eastern Memorial Hospital, New Taipei City, Taiwan, 2 Division of Nephrology, Department of Internal Medicine, National Taiwan University Hospital, National Taiwan University College of Medicine, Taipei, Taiwan

* taan70@yahoo.com.tw

\section{Abstract}

\section{G open access}

Citation: Chen HY, Chiu YL, Hsu SP, Pai MF, Yang JY, Peng YS (2016) Relationship between Fetuin A, Vascular Calcification and Fracture Risk in Dialysis Patients. PLoS ONE 11(7): e0158789. doi:10.1371/ journal.pone. 0158789

Editor: Martijn van Griensven, Klinikum rechts der Isar-Technical University Munich-TUM, GERMANY

Received: February 17, 2016

Accepted: June 22, 2016

Published: July 11, 2016

Copyright: @ 2016 Chen et al. This is an open access article distributed under the terms of the Creative Commons Attribution License, which permits unrestricted use, distribution, and reproduction in any medium, provided the original author and source are credited.

Data Availability Statement: All relevant data are within the paper and its Supporting Information files.

Funding: This study was supported by grants from Far Eastern Memorial Hospital (FEMH) (FEMH-95 C-025, FEMH-2011-C-006, FEMH-2012-D-032, FEMH-2014-D-021, FEMH-2015-D-034), Taiwan.

Competing Interests: The authors have declared that no competing interests exist.

Abbreviations: BMD, bone mineral density; BMI, body mass index; CaxP, calcium phosphate product; $\mathrm{CKD}$, chronic kidney disease; CV, cardiovascular;

\section{Background}

Fractures are a common morbidity that lead to worse outcomes in dialysis patients. Fetuin A inhibits vascular calcification (VC), potentially promotes bone mineralization and its level positively correlates with bone mineral density in the general population. On the other hand, the presence of $\mathrm{VC}$ is associated with low bone volume in dialysis patients. Whether the fetuin $\mathrm{A}$ level and $\mathrm{VC}$ can predict the occurrence of fractures in dialysis patients remains unknown.

\section{Methods}

We performed this prospective, observational cohort study including 685 dialysis patients (629 hemodialysis and 56 peritoneal dialysis) from a single center in Taiwan for a median follow-up period of 3.4 years. The baseline fetuin A level and status of presence of aortic arch calcification (VC) and incidence of major fractures (hip, pelvis, humerus, proximal forearm, lower leg or vertebrae) were assessed using adjusted Cox proportional hazards models, recursive partitioning analysis and competing risk models.

\section{Results}

Overall, 177 of the patients had major fractures. The incidence rate of major fractures was 3.29 per 100 person-years. In adjusted analyses, the patients with higher baseline fetuin $A$ levels had a lower incidence of fractures (adjusted hazard ratio (HR), 0.3; $95 \% \mathrm{Cl}, 0.18-0.5$, fetuin A tertile $3 v$ s. tertile 1 and $\mathrm{HR}, 0.52 ; 95 \% \mathrm{Cl}, 0.34-0.78$, tertile 2 vs. tertile 1$)$. The presence of aortic arch calcification (VC) independently predicted the occurrence of fractures (adjusted $\mathrm{HR}, 1.95 ; 95 \% \mathrm{Cl}, 1.34-2.84$ ) as well. When accounting for death as an event in competing risk models, the patients with higher baseline fetuin $\mathrm{A}$ levels remained to have a lower incidence of fractures (SHR, $0.31 ; 95 \% \mathrm{Cl}, 0.17-0.56$, fetuin A tertile $3 \mathrm{vs}$. tertile 1 and $0.51 ; 95 \% \mathrm{Cl}, 0.32-0.81$, tertile 2 vs. tertile 1$)$. 
$\mathrm{DM}$, diabetes mellitus; GNRI, geriatric nutritional risk index; HD, hemodialysis; HR, hazard ratio; hs-CRP, high sensitivity C-reactive protein; IQR, interquartile range; $\mathrm{PD}$, peritoneal dialysis; $\mathrm{PTH}$, intact parathyroid hormone; $\mathrm{VC}$, vascular calcification.

\section{Interpretations}

Lower baseline fetuin A levels and the presence of VC were independently linked to higher risk of incident fractures in prevalent dialysis patients.

\section{Introduction}

Patients with chronic kidney disease (CKD), especially those undergoing dialysis, have unique mineral and endocrine disturbances which result in altered bone structure and function. It has been shown that patients undergoing dialysis have higher rates of bone fractures compared to the general population.[1, 2] In addition, patients experiencing a major bone fracture (e.g. hip fracture) have been reported to have a remarkable increase in subsequent disability, death and hospitalization. [3-5] Several major risk factors such as abnormal intact parathyroid hormone (iPTH) levels, heavy comorbidity burden, sarcopenia, increased susceptibility to falls, and polypharmacy can increase the likelihood of fractures in dialysis patients. [2, 6, 7] However, the link between fractures and vascular calcification (VC), another major component of mineral bone disorders in CKD patients, is as yet uncertain. In CKD and dialysis patients, VC has been shown to correlate with low trabecular bone volume and indices of low bone turnover. [8,9] Adragao et al described an association between low bone volume and coronary calcifications in patients who were on dialysis for more than 6 years.[10] Of note, $\mathrm{VC}$ has a strong correlation with low bone volume in CKD patients, however little is known about the interrelationship between $\mathrm{VC}$ and fractures in dialysis patients.

Fetuin A is a glycoprotein synthesized in the liver and expressed in the extracellular space. It is a well-known inhibitor of $\mathrm{VC}$ in dialysis patients,[11] and it has been reported to promote bone mineralization in vitro. $[12,13]$ The role of fetuin $\mathrm{A}$ in tissue mineralization serving as a "mineral chaperone" has been proposed[14, 15]. In a landmark study, fetuin A colocalizes with matrix vesicles (MVs) which are secreted by human vascular smooth muscle cells (VSMCs) and is specifically loaded into MVs. These findings further strengthen the concerns between fetuin A and VC intracellularly[16]. However, its role in bone mineralization is understudied. In fetuin A knock-out mice, the trabecular bone mass and microstructure of cortical bone are unaffected by the absence of fetuin A; nevertheless, there is excess mineralization of the growth plate of long bone which causes short limbs[17]. In humans clinical study, the relationship between serum fetuin A level and bone mineral density (BMD) was investigated in 3075 wellfunctioning elderly persons, and the results showed that higher fetuin A levels were independently associated with higher BMD among women.[18] Nevertheless, a subsequent study showed no evidence of an association between fetuin A and the risk of clinical fractures.[19] These interesting but inconsistent findings prompted us to conduct this study in dialysis patients to investigate the connection between VC and bone volume and the potential link between the fetuin A and the risk of fractures as well. Therefore, the aim of this prospective, observational study was to test the hypothesis that dialysis patients with either lower fetuin A levels or VC would have a higher risk of incident fractures.

\section{Materials and Methods \\ Subjects}

This was a prospective study performed using five pooled patient cohorts. The first three cohorts were composed of 370, 238, and 216 prevalent hemodialysis (HD) patients, respectively, the fourth was composed of $220 \mathrm{HD}$ patients and 63 peritoneal dialysis (PD) patients 
and the fifth was $209 \mathrm{HD}$ patients. These patient cohorts have been described previously in more detail. [20-23] In brief, the five cohorts were collected prospectively to understand the associations between serum fetuin A level, inflammatory markers (such as, high sensitivity Creactive protein (hs-CRP) and lipid profiles with specific outcomes in prevalent dialysis patients, at the Far Eastern Memorial Hospital from 2007 to 2014. All patients had baseline data on fetuin A at entry. The exclusion criteria of the five cohorts were as follows: (1) active infection; (2) recent hospitalization within 3 months; (3) psychotic illness or other communication problems; (4) active malignancy; (5) younger than 20 years; (6) receiving HD or PD for less than 3 months; and (7) patients' refusal. In the flow diagram, we have clearly shown the reasons of exclusion in this cohort study (Fig 1). Before initiating this prospective study, we have re-evaluated all the participants in the five cohorts about the wills of being analyzed for the pre-specified outcome (fracture). For the most part, the exclusion of participants was due to repeated enrollment (88\%), only 14 patients (1\%) declined to participate this prospective

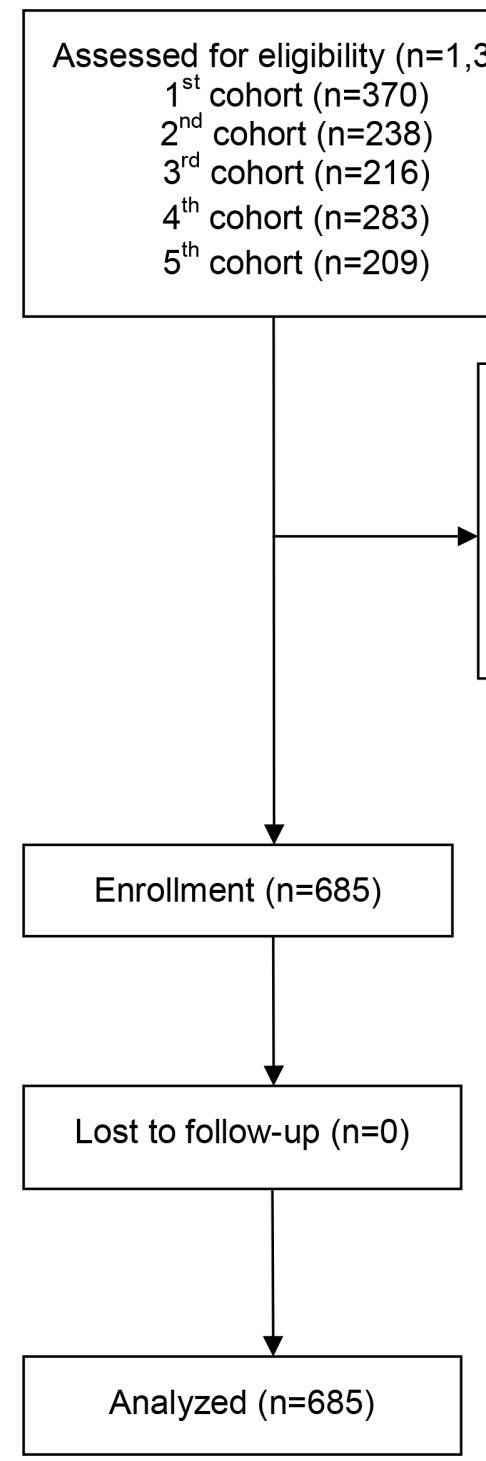

Excluded $(n=631)$

- Repeated enrollment $(n=557)$

- Other reasons $(n=60)$

- Lack of hs-CRP data $(n=28)$

- Lack of annual plain chest X-rays data $(n=32)$

- Declined to participate $(n=14)$

Fig 1. Study flow diagram.

doi:10.1371/journal.pone.0158789.g001 
analysis and none of the participants in the cohort were excluded because of missing fetuin A data. The final numbers of enrolled participants from the five cohorts are 354, 18, 117, 163 and 33 respectively.

All of the subjects provided written informed consent, the study complied with the World Medical Association Declaration of Helsinki-Ethical Principles for Medical Research Involving Human Subjects, and the Ethics Committee of Far Eastern Memorial Hospital approved the study protocol (ClinicalTrials.gov; NCT01457625).

In total, 685 patients (mean age, $59 \pm 13$ years; 348 women) who underwent prevalent $\mathrm{HD}$ (629) and PD (56) at the Far Eastern Memorial Hospital, Taiwan, were enrolled from February 2007 (first cohort), March 2009 (second cohort), March 2011 (third cohort), March 2013 (fourth cohort) and September 2014 (fifth cohort). The median dialysis vintage before recruitment was 2.5 years (range, $0.4-26.5$ years).

\section{Measurement of serum fetuin A concentrations}

Serum fetuin A levels in the five cohorts were measured using three types of highly sensitive, two-site enzyme-linked immunoassays (GenWay Biotech, Inc., San Diego, CA, USA; Adipo Bioscience, Inc., Santa Clara, CA, USA and R\&D Systems, Inc., Minneapolis, MN, USA). The intra-assay coefficients of variation were $4.1 \%, 4.0 \%$ and $4.0 \%$, respectively, and the inter-assay coefficients of variation were $6.2 \%, 6 \%$ and $6.4 \%$, respectively. The linear measurement ranges of the assays for human fetuin A levels were 0.002-2.5 g/L and 0.003-2.5 g/L and 0.002-2.5 g/L, respectively. Blood samples for the measurement of fetuin A levels were obtained once on recruitment and were immediately centrifuged and stored at $-70^{\circ} \mathrm{C}$ until the time of the assay.

\section{Measurements of clinical parameters}

Demographic data, a concurrent medical history of $\mathrm{CV}$ disease and smoking status were recorded. Venous blood was sampled in the morning after an overnight fast of more than 8 hours before the patient's mid-week dialysis session in the HD patients, or before the first daily dwell of dialysate in the PD patients. Intact PTH levels were determined by immunoassay (Roche Modular E170 analyzer). The hs-CRP levels were determined using the immunonephelometric method using a Tina-quant CRP (Latex) ultrasensitive assay (D \& P Modular Analyzer, Roche Diagnostics GmbH, Mannheim, Germany). The geriatric nutritional risk index (GNRI) was calculated by the following formula: GNRI $=[14.89 \times$ albumin level $(\mathrm{g} / \mathrm{dL})]+[41.7 \times$ body weight $/ \mathrm{WLo}]$, where WLo is the ideal body weight calculated from the Lorentz equation. The GNRI has previously been validated in dialysis patients, and a higher GNRI indicates better nutritional status.[24]

\section{Outcomes}

The primary outcome was the incidence of major fractures, which was defined as a new symptomatic fracture of the hip, pelvis, humerus, distal forearm, lower leg or vertebrae that occurred during follow-up. The occurrence of a major fracture was assessed by a clinical diagnosis (either from inpatient chart review or outpatient medical records), and concurrent roentgenogram, ordered for a suspicious fracture, with defined evidence of a fracture in the formal roentgenogram report by a radiologist. The outcome information was centrally assessed by trained clinicians, nephrologists and radiologists.

Follow-up started from February 2007 (first cohort entry), March 2009 (second cohort entry), March 2011 (third cohort entry), March 2013 (fourth cohort entry), and September 2014 (fifth cohort entry) and censored on the date of a major fracture, at the end of the study (July 31,2015), on the date of death or renal transplantation or at the time the patients were transferred to other dialysis facilities and were no longer followed up, whichever came first. 


\section{Assessment of VC}

We defined the presence of $\mathrm{VC}$ as the presence of aortic arch calcification on a posterior-anterior plain chest X-ray at the entry of study. All of the participants received routine annual posterior-anterior plain chest X-rays in our hospital. Two trained physicians blinded to the patients' clinical data reviewed plain chest X-rays performed before study enrollment to assess the presence of aortic arch calcification.

\section{Statistical analysis}

Continuous data were presented as mean \pm SD or median (interquartile range (IQR)), and categorical data were reported as percentages. Differences in baseline characteristics and biochemical parameters between the HD and PD patients and subjects with/without VC were compared using the Student's $t$ test and Mann-Whitney U test. Similarly, differences in baseline characteristics and biochemical parameters among the patients within the fetuin A tertiles were compared using ANOVA and the Kruskal-Wallis $\mathrm{H}$ test, as appropriate. The chi-square test was used for categorical variables.

Since the fetuin A level was not normally distributed in the dialysis patients $(\mathrm{P}<0.001$ by either Kolmogorov-Smirnov or Shapiro-Wilk Test), we constructed plots of the fetuin A levels and crude hazard ratios (HRs) of the incident major fractures using the Lowess function. The results revealed a non-linear relationship, suggesting the need for stratification of the patients into tertiles according to their fetuin A level for outcome analysis, which we then performed (S1 Fig). The primary predictor variables were the fetuin A level in each tertile: patients with a fetuin A level between 0.11-0.35 g/L were in tertile 1, between 0.35-0.65 g/L in tertile 2 and between $0.66-1.89 \mathrm{~g} / \mathrm{L}$ in tertile 3 .

Owing to the non-linear relationship between the fetuin A levels and HRs of the incident major fractures, we performed the outcome analysis in two ways: (1) to construct an algorithm for stratifying the major fracture risks in dialysis patients with recursive partitioning analysis (RPA) [25]. We performed the RPA in order to repeatedly divide patients into subgroups whether they had major fracture or not. It ideally provided a nonparametric discriminating tree for discriminating the power of risk factors of major fracture. Once RPA selected the tree, we only selected each of the splits identified with statistical criterion of $P<0.01$ for outcomes (incident major fracture). Any split that did not meet this criterion was deleted. The final nodes were then compared. Kaplan-Meier graphs were presented for the final set of prognostic groups; (2) and standard Cox proportional hazard models. We used the "Enter" method to analyze the HR of each primary predictor variable in the model. For each fracture outcome, we first adjusted for gender, age, dialysis vintage, previous fracture history, diabetes mellitus (DM) status/hypertension status, dialysis modality, smoking/alcohol status and patient cohort (Model 1). Additional models were further adjusted for factors potentially associated with the risk of fractures that may have been confounders of the association between fetuin A level and fractures. Model 2 was adjusted for the covariates in Model 1 as well as hemoglobin level, nutritional status (GNRI), iPTH, calcium phosphate product (CaXP) and hs-CRP level. In Model 3, we also adjusted for the presence of aortic arch calcification. Sensitivity analyses were performed to test the robustness of our findings. Analyses of fracture outcome were performed while accounting for the competing event of death using the method of Fine and Gray[26], because death may be an informative censoring event. Owing to the pathophysiological link between fetuin A and VC, we further explored the potential interaction between fetuin A and VC in the prediction of incident fractures. All of the statistical analyses were performed using SPSS software version 19.0 (SPSS, Inc., Chicago, IL, USA) and Stata IC, version 14 (StataCorp, College Station, TX). A P value less than 0.05 was considered to be statistically significant. 
Table 1. Baseline characteristics of the all patients and the patients by fetuin A tertile.

\begin{tabular}{|c|c|c|c|c|c|}
\hline & All patients & Fetuin $\mathrm{A}$ tertile $1(0.11-0.35)$ & Fetuin A tertile $2(0.35-0.65)$ & Fetuin A tertile $3(0.66-1.89)$ & P value \\
\hline & $n=685$ & $n=228$ & $n=229$ & $n=228$ & \\
\hline Age (years) & $59 \pm 13$ & $61 \pm 12$ & $59 \pm 13$ & $56 \pm 13$ & 0.001 \\
\hline Female gender (\%) & 51 & 49 & 52 & 52 & 0.7 \\
\hline Diabetes mellitus (\%) & 47 & 51 & 47 & 44 & 0.3 \\
\hline Dialysis vintage (years) & $2.5(1.5,4.4)$ & $2.6(1.9,4.6)$ & $2.4(1.6,4.6)$ & $2.5(1.3,4.2)$ & 0.1 \\
\hline History of hypertension (\%) & 78 & 81 & 76 & 78 & 0.4 \\
\hline History of previous fracture (\%) & 16 & 21 & 14 & 14 & 0.2 \\
\hline Systolic BP (mmHg) & $144 \pm 31$ & $145 \pm 31$ & $146 \pm 42$ & $146 \pm 72$ & 0.3 \\
\hline Diastolic BP $(\mathrm{mmHg})$ & $84 \pm 12$ & $84 \pm 13$ & $86 \pm 18$ & $83 \pm 14$ & 0.2 \\
\hline $\mathrm{BMI}\left(\mathrm{kg} / \mathrm{m}^{2}\right)$ & $23.1 \pm 3.9$ & $22.3 \pm 3.9$ & $23.3 \pm 3.8$ & $23.8 \pm 3.8$ & $<0.001$ \\
\hline \multicolumn{6}{|l|}{ Laboratory data } \\
\hline Hemoglobin (g/dL) & $11.0 \pm 1.4$ & $10.9 \pm 1.5$ & $11.1 \pm 1.4$ & $10.9 \pm 1.4$ & 0.4 \\
\hline Cre $(\mathrm{mg} / \mathrm{dL})$ & $10.9 \pm 2.4$ & $10.6 \pm 2.2$ & $10.9 \pm 2.4$ & $11.1 \pm 2.7$ & 0.1 \\
\hline $\mathrm{K}(\mathrm{mmol} / \mathrm{L})$ & $4.7 \pm 0.8$ & $4.8 \pm 0.7$ & $4.6 \pm 0.8$ & $4.6 \pm 0.8$ & 0.1 \\
\hline $\mathrm{Ca}(\mathrm{mg} / \mathrm{dL}) ;$ corrected & $9.2 \pm 0.7$ & $9.1 \pm 0.7$ & $9.3 \pm 0.7$ & $9.3 \pm 0.7$ & 0.01 \\
\hline $\mathrm{P}(\mathrm{mg} / \mathrm{dL})$ & $5.3 \pm 1.4$ & $5.2 \pm 1.4$ & $5.2 \pm 1.3$ & $5.4 \pm 1.4$ & 0.5 \\
\hline CaxP & $49 \pm 14$ & $48 \pm 13$ & $48 \pm 13$ & $50 \pm 14$ & 0.2 \\
\hline iPTH (pg/mL) & $257(123,485)$ & $254(130,450)$ & $298(125,580)$ & $240(114,463)$ & 0.5 \\
\hline hs-CRP (mg/L) & $3.1(1.1,7.6)$ & $3.4(1.1,8.4)$ & $3.0(1.1,7.3)$ & $2.9(1.2,7.2)$ & 0.5 \\
\hline Albumin (g/L) & $4.1 \pm 0.4$ & $4.0 \pm 0.4$ & $4.1 \pm 0.4$ & $4.1 \pm 0.4$ & 0.3 \\
\hline GNRI & $104.3 \pm 9.6$ & $102.3 \pm 10$ & $104.5 \pm 8.9$ & $106.1 \pm 9.6$ & $<0.001$ \\
\hline \multicolumn{6}{|l|}{ Medications (\%) } \\
\hline ESA & 91 & 91 & 91 & 90 & 0.3 \\
\hline Active Vitamin D3 & 45 & 46 & 43 & 45 & 0.3 \\
\hline Phosphorus binder & 86 & 87 & 84 & 87 & 0.2 \\
\hline Calcium-containing & 64 & 64 & 65 & 63 & 0.1 \\
\hline Non-calcium-containing & 22 & 23 & 19 & 24 & 0.2 \\
\hline Medications for osteoporosis & 0.5 & 0.4 & 0.8 & 0.4 & 0.3 \\
\hline Anti-hypertensive agents & 55 & 55 & 60 & 52 & 0.2 \\
\hline
\end{tabular}

Abbreviations: CVD, cardiovascular disease; BP, blood pressure; Cre, creatinine; BMI, body mass index; CaxP, calcium phosphate product; iPTH, intact parathyroid hormone; hs-CRP, high-sensitive C-reactive protein; GNRI, geriatric nutritional risk index; ESA, erythropoiesis-stimulating agents. Note: Conversion factors for units: hemoglobin in $\mathrm{g} / \mathrm{dL}$ to $\mathrm{g} / \mathrm{L}, \times 10$; serum calcium in $\mathrm{mg} / \mathrm{dL}$ to $\mathrm{mmol} / \mathrm{L}, \times 0.2495$; serum phosphate in $\mathrm{mg} / \mathrm{dL}$ to $\mathrm{mmol} / \mathrm{L}$, $\times 0.3229$; serum albumin in $\mathrm{g} / \mathrm{dL}$ to $\mathrm{g} / \mathrm{L}, \times 10$. No conversion is necessary for serum iPTH in $\mathrm{pg} / \mathrm{mL}$ and $\mathrm{ng} / \mathrm{L}$; serum potassium in $\mathrm{mEq} / \mathrm{L}$ and $\mathrm{mmol} / \mathrm{L}$.

\section{Results}

\section{The baseline characteristics of the participants}

The baseline characteristics of all of the participants, those in the fetuin A tertiles are summarized in Table 1. The patients in the fetuin A tertiles had different age, body mass index (BMI) and nutritional status (Table 1). And the baseline characteristics of the participants receiving $\mathrm{HD}$ or PD and with/without VC are summarized in the Table 2. In general, more female patients received $\mathrm{PD}$, fewer patients undergoing $\mathrm{PD}$ had diabetes, and those undergoing $\mathrm{PD}$ were younger, had lower hemoglobin and albumin levels, higher BMI, creatinine and fetuin A levels (Table 2). Patients with aortic arch calcification were older, had longer dialysis vintage, had higher levels of hs-CRP and lower fetuin A levels (Table 2). 


\section{Outcomes}

Overall, 177 of the participants experienced incident major fractures during a median of 3.4 years (IQR, 1.5-5.8 years) of follow-up. Thirty-seven participants had hip fractures, 10 had pelvic fractures, 20 had humeral fractures, 28 had distal forearm fractures, 32 had lower leg fractures and 50 had vertebral fractures. The incidence rate of major fractures was 3.29 per 100 person-years. The incidence rates in the lowest to highest fetuin A tertiles were 4.83, 3.21 and 1.45 per 100 person-years, respectively.

\section{Assessment of VC}

A total of 365 participants had aortic arch calcification on the plain chest X-rays with different severity at entry, including 154, 124 and 87 participants in fetuin A tertile 1, 2 and 3,

Table 2. Baseline characteristics of the patients undergoing hemodialysis (HD) and peritoneal dialysis (PD) and patients with and without vascular calcification (VC).

\begin{tabular}{|c|c|c|c|c|c|c|}
\hline & HD patients & PD patients & P value & Patients with VC & Patients without VC & P value \\
\hline & $n=629$ & $n=56$ & & $n=365$ & $n=320$ & \\
\hline Age (years) & $59 \pm 12$ & $53 \pm 13$ & 0.001 & $64 \pm 11$ & $53 \pm 12$ & $<0.001$ \\
\hline Female gender (\%) & 49 & 69 & 0.004 & 56 & 45 & 0.004 \\
\hline Diabetes mellitus (\%) & 50 & 27 & 0.001 & 56 & 38 & $<0.001$ \\
\hline Dialysis vintage (years) & $2.4(1.4,4.6)$ & $2.6(1.0,5.4)$ & 0.9 & $2.9(1.6,5.0)$ & $2.2(1.2,4.2)$ & 0.01 \\
\hline History of hypertension (\%) & 79 & 85 & 0.3 & 83 & 73 & 0.001 \\
\hline History of previous fracture (\%) & 21 & 31 & 0.1 & 20 & 14 & 0.08 \\
\hline Systolic BP (mmHg) & $146 \pm 43$ & $147 \pm 50$ & 0.1 & $147 \pm 50$ & $146 \pm 47$ & 0.2 \\
\hline Diastolic BP (mmHg) & $85 \pm 13$ & $83 \pm 19$ & 0.2 & $84 \pm 20$ & $82 \pm 16$ & 0.1 \\
\hline BMI $\left(\mathrm{kg} / \mathrm{m}^{2}\right)$ & $23.0 \pm 3.9$ & $23.9 \pm 3.1$ & 0.02 & $23.3 \pm 4.0$ & $22.9 \pm 3.7$ & 0.2 \\
\hline \multicolumn{7}{|l|}{ Laboratory data } \\
\hline Hemoglobin (g/dL) & $11.0 \pm 1.4$ & $10.5 \pm 1.2$ & 0.006 & $11.1 \pm 1.4$ & $10.9 \pm 1.4$ & 0.05 \\
\hline Cre $(\mathrm{mg} / \mathrm{dL})$ & $10.7 \pm 3.8$ & $11.3 \pm 3.2$ & 0.02 & $10.8 \pm 2.9$ & $11.0 \pm 3.4$ & 0.4 \\
\hline $\mathrm{K}(\mathrm{mmol} / \mathrm{L})$ & $4.7 \pm 0.8$ & $4.1 \pm 0.7$ & 0.07 & $4.7 \pm 0.8$ & $4.6 \pm 0.8$ & 0.08 \\
\hline $\mathrm{Ca}(\mathrm{mg} / \mathrm{dL}) ;$ corrected & $9.2 \pm 0.7$ & $9.3 \pm 0.7$ & 0.4 & $9.2 \pm 0.8$ & $9.2 \pm 0.7$ & 0.5 \\
\hline $\mathrm{P}(\mathrm{mg} / \mathrm{dL})$ & $5.3 \pm 1.4$ & $5.3 \pm 1.1$ & 0.8 & $5.2 \pm 1.4$ & $5.3 \pm 1.4$ & 0.3 \\
\hline CaxP & $49 \pm 14$ & $49 \pm 11$ & 0.8 & $48 \pm 14$ & $49 \pm 14$ & 0.4 \\
\hline iPTH (pg/mL) & $257(122,485)$ & $292(128,514)$ & 0.8 & $235(116,464)$ & $289(140,506)$ & 0.06 \\
\hline hs-CRP (mg/L) & $3.1(1.2,7.6)$ & $2.4(0.7,6.2)$ & 0.1 & $4.3(1.6,9.7)$ & $2.3(0.8,5.4)$ & $<0.001$ \\
\hline Albumin (g/L) & $4.1 \pm 0.4$ & $3.9 \pm 0.3$ & 0.01 & $4.0 \pm 0.4$ & $4.1 \pm 0.4$ & 0.02 \\
\hline Fetuin $A(g / L)$ & $0.45(0.29,0.7)$ & $0.79(0.65,1.21)$ & $<0.001$ & $0.4(0.27,0.64)$ & $0.62(0.37,0.82)$ & $<0.001$ \\
\hline GNRI & $104.4 \pm 9.7$ & $102.8 \pm 8.0$ & 0.2 & $103.9 \pm 9.7$ & $104.7 \pm 9.5$ & 0.2 \\
\hline \multicolumn{7}{|l|}{ Medications (\%) } \\
\hline ESA & 91 & 90 & 0.3 & 92 & 89 & 0.1 \\
\hline Active Vitamin D3 & 45 & 48 & 0.6 & 44 & 49 & 0.1 \\
\hline Phosphorus binder & 85 & 90 & 0.4 & 90 & 84 & 0.5 \\
\hline Calcium-containing & 64 & 67 & 0.5 & 60 & 54 & 0.3 \\
\hline Non-calcium-containing & 21 & 23 & 0.3 & 30 & 30 & 0.4 \\
\hline Medications for osteoporosis & 0.4 & 1.7 & 0.06 & 0.5 & 0.5 & 0.9 \\
\hline Anti-hypertensive agents & 60 & 52 & 0.2 & 55 & 57 & 0.2 \\
\hline
\end{tabular}

Abbreviations: CVD, cardiovascular disease; BP, blood pressure; Cre, creatinine; BMI, body mass index; CaxP, calcium phosphate product; iPTH, intact parathyroid hormone; hs-CRP, high-sensitive C-reactive protein; GNRI, geriatric nutritional risk index; ESA, erythropoiesis-stimulating agents. Note: Conversion factors for units: hemoglobin in $\mathrm{g} / \mathrm{dL}$ to $\mathrm{g} / \mathrm{L}, \times 10$; serum calcium in $\mathrm{mg} / \mathrm{dL}$ to $\mathrm{mmol} / \mathrm{L}, \times 0.2495$; serum phosphate in $\mathrm{mg} / \mathrm{dL}$ to $\mathrm{mmol} / \mathrm{L}$, $\times 0.3229$; serum albumin in $\mathrm{g} / \mathrm{dL}$ to $\mathrm{g} / \mathrm{L}, \times 10$. No conversion is necessary for serum $\mathrm{PTH}$ in $\mathrm{pg} / \mathrm{mL}$ and $\mathrm{ng} / \mathrm{L}$; serum potassium in $\mathrm{mEq} / \mathrm{L}$ and $\mathrm{mmol} / \mathrm{L}$. doi:10.1371/journal.pone.0158789.t002 
Table 3. Hazard ratios (HRs) of fetuin A tertiles in predicting the occurrence of major fractures using different Cox proportional hazard regression models.

\begin{tabular}{l|l|l|l}
\hline Variables & Model 1 & Model 2 & Model 3 \\
\hline & Adjusted HR(95\% Cl) & Adjusted HR(95\% Cl) & Adjusted HR(95\% Cl) \\
\hline Fetuin A tertiles & & & \\
\hline Fetuin A tertiles (3 ${ }^{\text {rd }}$ vs. $1^{\text {st }}$ tertile) & $0.28(0.17-0.47)$ & $0.30(0.18-0.50)$ & $0.34(0.2-0.57)$ \\
\hline Fetuin A tertiles (2 ${ }^{\text {nd }}$ vs. $1^{\text {st }}$ tertile) & $0.54(0.36-0.81)$ & $0.52(0.34-0.78)$ & $0.53(0.34-0.81)$ \\
\hline Other variables & & & \\
\hline Gender (male vs. female) & $0.68(0.58-0.92)$ & $0.68(0.49-0.94)$ & $0.72(0.52-0.99)$ \\
\hline Age (every 1 year older) & $1.03(1.01-1.04)$ & $1.03(1.01-1.04)$ & $1.02(1.002-1.03)$ \\
\hline iPTH (every 100 unit increase) & - & $1.05(1.01-1.1)$ & $1.04(1.008-1.12)$ \\
\hline hs-CRP (every 1 unit increase) & - & $1.10(1.02-1.18)$ & $1.11(1.02-1.19)$ \\
\hline Aortic arch calcification (yes vs. no) & - & - & $1.95(1.34-2.84)$ \\
\hline
\end{tabular}

Abbreviations: $\mathrm{HR}$, hazard ratio; $\mathrm{Cl}$, confidence interval; iPTH, intact parathyroid hormone; hs-CRP, high-sensitive C-reactive protein, GNRI, geriatric nutritional risk index

${ }^{\S}$ Adjusted for model 1: gender, age, dialysis vintage, previous fracture history, diabetes status/hypertension status, dialysis modality, smoking/alcohol status and patient cohort; model 2: factors in model 1 and hemoglobin level, intact parathyroid hormone, GNRI, CaxP and high-sensitive C-reactive protein (hsCRP) levels; model 3: factors in model 2 and presence of aortic arch calcification.

respectively. The number of participants with aortic arch calcification was significantly different in the fetuin $\mathrm{A}$ tertiles $(\mathrm{P}<0.001$ by the chi-square test). One hundred and twenty-eight subjects with aortic arch calcification and 49 subjects without aortic arch calcification had major fractures. The incidence rates of major fractures in the participants with and without aortic arch calcification were 4.29 and 1.87 per 100 person-years, respectively.

\section{Associations between fetuin A level and fractures}

In the multivariate Cox regression model, patients with higher fetuin A levels had a lower incidence of major fracture (adjusted HR, $0.28 ; 95 \%$ CI, 0.17-0.47, tertile $3 v$ s. tertile 1; adjusted HR, $0.54 ; 95 \%$ CI, $0.36-0.81$, tertile 2 vs. tertile 1 ) in Model 1 . Similarly, patients with higher fetuin A levels had a lower incidence of fractures in Model 2 and 3 (Table 3). Patients with aortic arch calcification at study entry had a higher risk of incident fractures (adjusted HR, 1.95; 95\% CI, 1.34-2.84). Fetuin A levels and the presence of VC both independently predicted the risk of major fracture in our dialysis patients.

\section{Recursive partitioning analysis}

As we have shown in the analysis from the Cox proportional hazards model, fetuin A tertiles, gender, age, iPTH, hs-CRP and VC were among the significant predictive factors (Table 3 ) and they have selected to divide the patient population.

Two hundred and twenty-eight patients were in the tertile 3 groups. For the patients in this group, presence of VC indicated a higher incidence of fracture, and among those within fetuin A tertile $2(\mathrm{~N}=229)$, presence of $\mathrm{VC}$ indicated a higher incidence of fracture as well. However, the subjects in the fetuin A tertile 1, presence of VC did not further differentiate the risk of major fracture (Fig 2). The 5 groups were ultimately defined (Node 1: within fetuin A tertile 1, with or without VC; node 4: within fetuin A tertile 2, without VC; node 5: within fetuin A tertile 2, with VC; node 6: within fetuin A tertile 3, without VC and node 7: within fetuin A tertile 3, with VC), and the Kaplan-Meier curves for these 5 groups are shown in Fig 3. Patients within the fetuin A tertile 3 and without VC had the lowest incidence of major fracture (Node 6). 


\section{Associations between fetuin A level and VC}

Because of the pathophysiological link between fetuin A and VC, as well as the very much interaction between fetuin A and VC (Fig 2), we further explored the interaction between fetuin $\mathrm{A}$ and $\mathrm{VC}$ in the prediction of incident fractures. We reported the interaction between

\section{Major fracture}

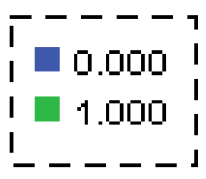

\begin{tabular}{|lccc|}
\hline \multicolumn{4}{|c|}{ Node 0} \\
Category & $\%$ & $\mathrm{~N}$ \\
\hline 0.000 & 74.2 & 508 \\
1.000 & 25.8 & 177 \\
\hline Total & 100.0 & 685 \\
\hline & &
\end{tabular}

Fetuin Atertiles

A.dj. $\mathrm{F}=0.000$, Chi-

square=45.445, df $=2$

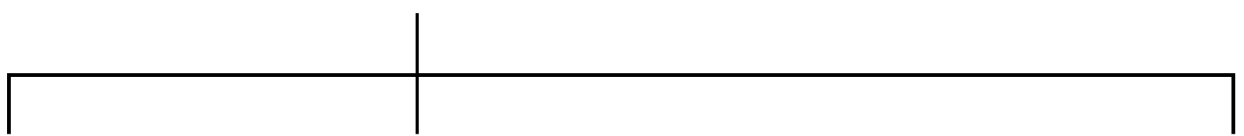

Fetuin A tertile 1

\begin{tabular}{|llll|}
\hline \multicolumn{3}{|c|}{ Node 1} \\
Category & $\%$ & $\mathrm{~N}$ \\
\hline 0.000 & 60.5 & 139 \\
1.000 & 39.5 & 90 \\
\hline Total & 33.3 & 229 \\
\hline
\end{tabular}

Fetuin A tertile 2

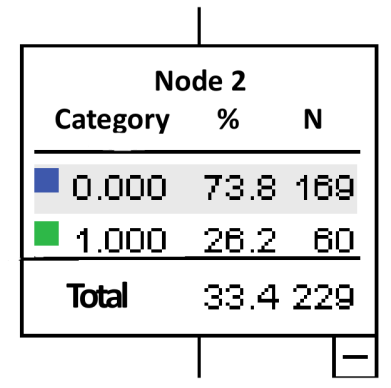

Vascular calcification

A.ji. $F=0.002$, Chi-

square=10.049, df=1
Fetuin A tertile 3

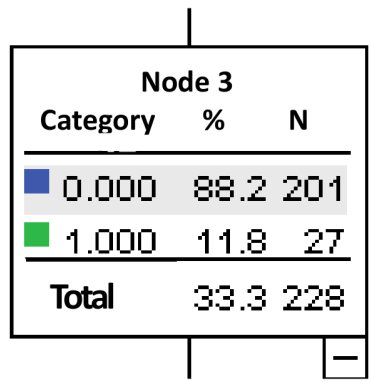

Vascular calcification

Adj. $\mathrm{P}=0.000$, Chisquare=16.742, df=1
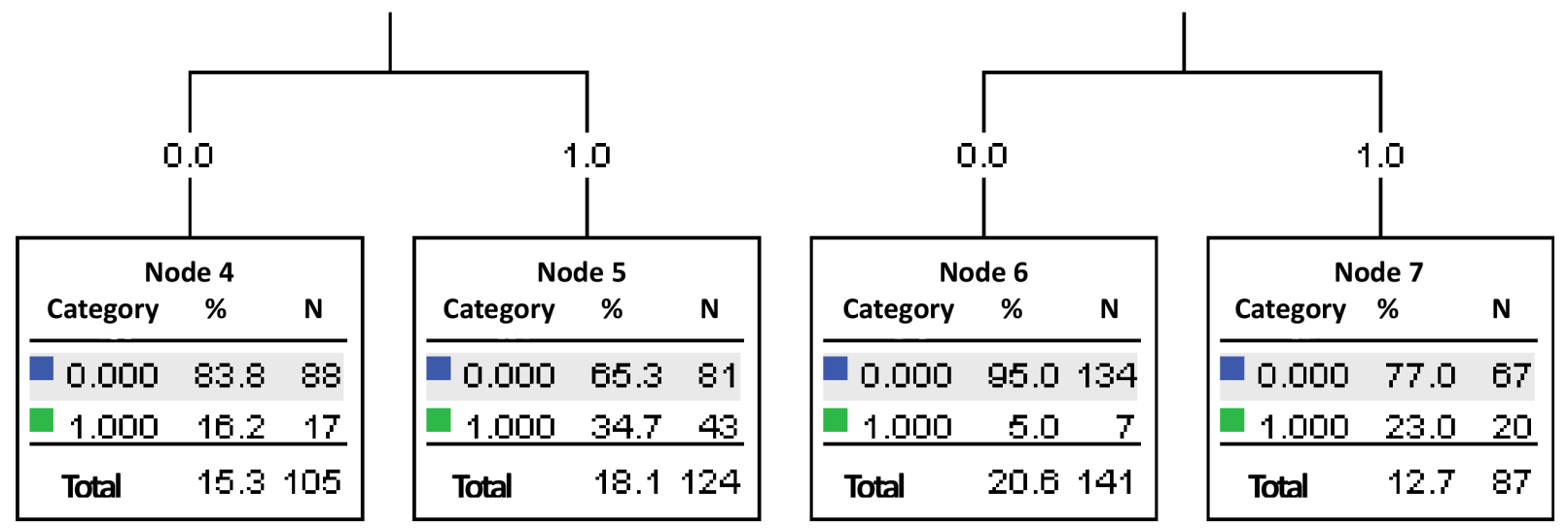

Fig 2. Classification tree based on recursive partitioning analysis.

doi:10.1371/journal.pone.0158789.g002 


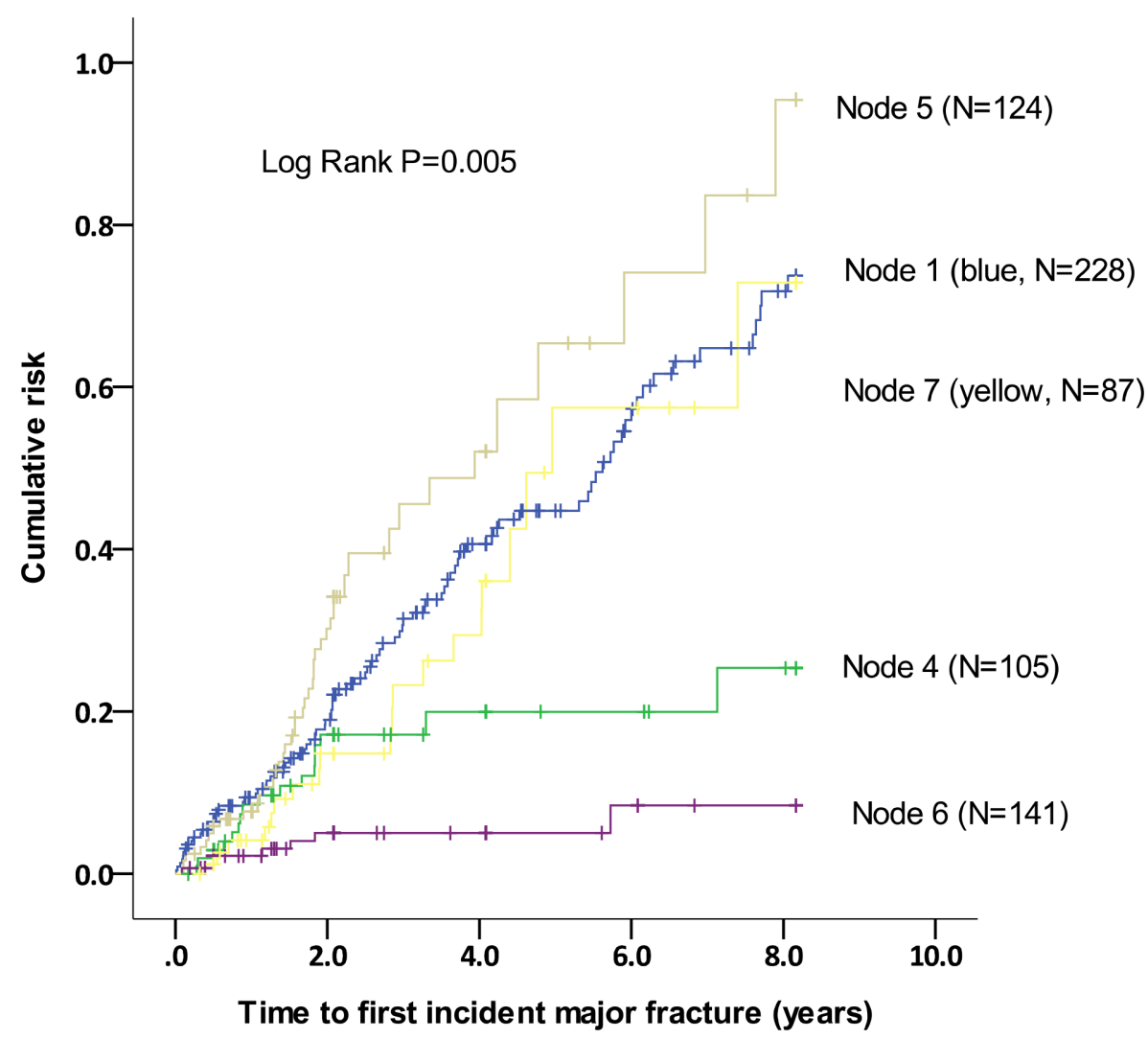

Fig 3. Kaplan-Meier survival curves for all patients, by risk group. Node 1: within fetuin $A$ tertile 1, with or without VC; node 4: within fetuin A tertile 2, without VC; node 5: within fetuin A tertile 2, with VC; node 6: within fetuin A tertile 3, without VC and node 7: within fetuin A tertile 3, with VC.

doi:10.1371/journal.pone.0158789.g003

fetuin A tertile and the presence of aortic arch calcification on the risk of incident major fracture in the Table 4. Either in patients with or without aortic arch calcification, those with higher fetuin A levels (tertile 2 and 3 ) had lower risk of incident fracture (P for interaction $=0.08$, Table 4 ).

\section{Comparison between PD and HD patients}

Compared with PD patients, HD patients had lower fetuin A concentrations (0.47, 0.29- 0.7 vs. $0.79,0.65-1.21 \mathrm{~g} / \mathrm{L}, \mathrm{P}<0.001$ ) (Table 2). The incidence rates of major fractures in our $\mathrm{PD}$ and HD patients were 0.43 and 3.41 per 100 person-years, respectively. HD patients had a

Table 4. Interaction between fetuin A tertile and the presence of aortic arch calcification on the risk of incident major fracture.

\begin{tabular}{|c|c|c|c|c|c|c|c|c|}
\hline & \multicolumn{2}{|c|}{ Fetuin A tertile $1(\mathrm{~N}=228)$} & \multicolumn{2}{|c|}{ Fetuin A tertile $2(\mathrm{~N}=229)$} & \multicolumn{2}{|c|}{ Fetuin A tertile $3(\mathrm{~N}=228)$} & \multirow[b]{2}{*}{$\begin{array}{l}P \text { for } \\
\text { trend }\end{array}$} & \multirow[b]{2}{*}{$\begin{array}{l}\text { P for } \\
\text { interaction }\end{array}$} \\
\hline & $\begin{array}{l}\mathrm{N} \text { with/without } \\
\text { Major fracture }\end{array}$ & HR 95\% Cl & $\begin{array}{l}\mathrm{N} \text { with/without } \\
\text { Major fracture }\end{array}$ & HR $95 \% \mathrm{Cl}$ & $\begin{array}{l}\mathrm{N} \text { with/without } \\
\text { Major fracture }\end{array}$ & HR 95\%Cl & & \\
\hline $\begin{array}{l}\text { With aortic arch } \\
\text { calcification }(n=365)\end{array}$ & $65 / 89$ & 1.0 & $43 / 81$ & $\begin{array}{l}0.54(0.33- \\
0.87) \mathrm{P}=0.01\end{array}$ & $20 / 67$ & $\begin{array}{l}0.47(0.26- \\
0.99) P=0.05\end{array}$ & $P=0.03$ & $P=0.08$ \\
\hline $\begin{array}{l}\text { Without aortic arch } \\
\text { calcification }(n=320)\end{array}$ & $25 / 49$ & $\begin{array}{l}0.64(0.39- \\
1.04) \mathrm{P}=0.07\end{array}$ & $17 / 88$ & $\begin{array}{l}0.29(0.16- \\
0.55) P<0.001\end{array}$ & $7 / 134$ & $\begin{array}{l}0.11(0.05- \\
0.26) \mathrm{P}<0.001\end{array}$ & $P<0.001$ & \\
\hline
\end{tabular}

Adjusted for gender, age; dialysis vintage, diabetes/hypertension status, patient cohort, intact parathyroid hormone; body mass index, albumin and highsensitive C-reactive protein (hs-CRP) levels, fetuin A tertile and presence of aortic arch calcification

Abbreviations: $\mathrm{N}$, number; $\mathrm{HR}$, hazard ratio; $\mathrm{Cl}$, confidence interval

doi:10.1371/journal.pone.0158789.t004 
Table 5. Competing risk analysis of the relative hazard of major fracture by fetuin A tertile (death is the competing event).

\begin{tabular}{|c|c|c|c|}
\hline Variables & Model 1 & Model 2 & Model 3 \\
\hline & Adjusted SHR(95\% Cl) $\$$ & Adjusted SHR(95\% Cl) $)^{\S}$ & Adjusted SHR(95\% Cl) $\$$ \\
\hline \multicolumn{4}{|l|}{ Fetuin A tertiles } \\
\hline Fetuin $\mathrm{A}$ tertiles $\left(3^{\text {rd }} v s .1^{\text {st }}\right.$ tertile $)$ & $0.27(0.16-0.48)$ & $0.27(0.15-0.49)$ & $0.31(0.17-0.56)$ \\
\hline Fetuin A tertiles $\left(2^{\text {nd }} v s .1^{\text {st }}\right.$ tertile $)$ & $0.54(0.35-0.82)$ & $0.49(0.32-0.78)$ & $0.51(0.32-0.81)$ \\
\hline Aortic arch calcification (yes vs. no) & - & - & $1.88(1.28-2.77)$ \\
\hline
\end{tabular}

Abbreviations: SHR, subhazard ratio; $\mathrm{Cl}$, confidence interval; $\mathrm{iPTH}$, intact parathyroid hormone; hs-CRP, high-sensitive C-reactive protein, GNRI, geriatric nutritional risk index

${ }^{\S}$ Adjusted for model 1: gender, age, dialysis vintage, previous fracture history, diabetes status/hypertension status, dialysis modality, smoking/alcohol status and patient cohort; model 2: factors in model 1 and hemoglobin level, intact parathyroid hormone, GNRI, CaxP and high-sensitive C-reactive protein (hsCRP) levels; model 3: factors in model 2 and presence of aortic arch calcification.

doi:10.1371/journal.pone.0158789.t005

significant higher risk to have incident fracture compared with PD patients (adjusted HR, 4.95; 95\% CI, 1.19-20.68). In addition, the incidence rates of VC in our PD and HD patients were 3.49 and 6.78 per 100 person-years, respectively. Nonetheless, no significant difference was observed in the association of fetuin A level with the risk of major fractures between the two dialysis modalities (dialysis modality interaction analysis, $\mathrm{P}=0.8$ ).

\section{Sensitivity Analyses}

When accounting for death as a potential informative event in competing risk models, the subhazard ratio (SHR) for major fracture continued to be significantly less for dialysis patients with higher fetuin A level examined in a fully adjusted model as well as those without the presence of aortic arch calcification (Table 5).

\section{Discussion}

The main finding of this study is that the prevalent dialysis patients with a higher baseline fetuin A level had a lower long-term risk of incident major fractures regardless of gender, dialysis modality (HD or PD) and nutritional or inflammatory status; besides, patients with VC at study entry also had a higher risk to have incident fracture. Interestingly, the risk of incident fractures and aortic arch calcification declined in parallel with the increase in fetuin A level; however, lower fetuin A level and the presence of VC both independently predicted the higher risk of incident fracture in dialysis patients. In addition to the inhibitory property of $\mathrm{VC}$ and the strong predictive power of $\mathrm{CV}$ death, the fetuin A level also predicted the occurrence of major fractures in the dialysis patients.

Major fractures lead to high mortality and morbidity rates in dialysis patients, $[3,5]$ and therefore it is critical to identify high-risk patients who are susceptible to fragile fractures and to prevent potential fracture events in the long-term care of prevalent dialysis patients. Our results showed a high incidence of major fractures in the dialysis patients, around 3.29 per 100 person-years, which is nearly three times greater than that reported for elderly patients without CKD.[19] This incidence rate is similar to that presented in the Dialysis Outcomes and Practice Patterns Study [5] and the U.S. Renal Data System.[3] Of note, we found a link between fetuin A and incident fractures in our dialysis patients, that is, a higher fetuin A level predicted a lower incidence of fractures (Table 3, Fig 2). To the best of our knowledge, this is the first study to report an association between fetuin $\mathrm{A}$ level and incident fractures in dialysis patients. Therefore, fetuin A level in prevalent dialysis patients should be recognized as a potential marker to identify the dialysis patients who are at a higher risk of a major fracture. 
In a landmark study using bone biopsies to estimate bone volume and multi-slice computed tomography to assess coronary artery calcification, low bone volume was found to be a significant risk factor for coronary calcification in HD patients.[10] In addition, low bone volume has been recognized as an important risk factor for the occurrence of fractures in both the general population [27-29] and dialysis patients.[30, 31] Therefore, it is reasonable to assume that dialysis patients with evident VC are more likely to experience a fracture. Our results have clearly shown that the dialysis patients with aortic arch calcification had a higher risk of fractures (Table 3, Figs 2 and 3). Specifically, in uremic milieu, dialysis patients tended to consume more fetuin A to prevent VC and therefore, dialysis subjects with baseline VC tended to have a lower fetuin A level concurrently. In an outstanding study which has shown that fetuin A is trafficked and exocytosed via exosome release in MV bodies. Interestingly, MV bodies containing exosomes were easily observed in vessels, especially in calcified MVs in dialysis patients. Factors that increase exosome release will promote vascular calcification, specifically under the condition of environmental calcium stress or fetuin A deficiency[16]. Therefore, the consequent generalized VC can be highly anticipated.

The features of osteoporosis in CKD patients are low trabecular bone volume and disrupted micro-architecture, but no abnormalities in mineralization or bone turnover[32]. Instead, bone loss is mostly from cortical bone in subjects with CKD mineral and bone disorder (CKD-MBD), and their iPTH, alkaline phosphatase, Klotho, sclerostin, and fetuin A levels are pronouncedly altered[33]. Due to the high prevalence of osteoporosis and CKD-MBD in CKD subjects, both conditions are commonly existent simultaneously. But, in reality, the CKD$\mathrm{MBD}$ is more complex than osteoporosis and CKD-MBD influences bone quality, contributes to high rates of fracture and most importantly, it results in VC in CKD patients. Although they both result in bone fragility, they do have different pathophysiology to destroy the bone. Osteoporosis is induced by excessive osteoclastic bone resorption in postmenopausal woman and subjects with aging process. But CKD-MBD is related to altered mineral metabolism and the imbalance of pro- and anti-calcification factors (such as fetuin A deficiency) which induced either high or low turnover bone disease in CKD subjects [33]. The post hoc analyses of data from crucial osteoporosis studies suggest that in patients with mild stage $3 \mathrm{CKD}$ and normal $\mathrm{iPTH}$, calcium and phosphate levels, conventional treatments for osteoporosis (such as bisphosphonates, teriparatide......) are effective to reduce fracture rates. Nevertheless, for patients with stage $4-5 \mathrm{CKD}$, the available evidence are insufficient to determine whether these medications are effective $[33,34]$. Briefly speaking, low bone density and fractures induced by osteoporosis in patients with CKD differ from those with CKD-MBD.

In a recent investigation, Fink et al reported that serum fetuin A level had a positive association with areal BMD, but that there was no evidence of an association between fetuin $\mathrm{A}$ and the risk of clinical fractures in a large community cohort including 4714 elderly participants ( $>65$ years of age)(the Cardiovascular Health Study).[19] A similar study including an even older cohort (70-79 years of age), showed that a higher fetuin A level was correlated with higher BMD in older women.[18] Based on these reports, fetuin A level can predict BMD, but not the occurrence of major fractures in the elderly. These results seem to be in contrast to our findings; however, this may be due to several essential differences in the studies. First, our study was performed in patients undergoing dialysis rather than generally healthy elderly subjects. In the previous investigation, the dialysis patients had a lower serum fetuin A level than the ageand gender-matched cohort without CKD.[11] Our participants had a median level of fetuin A of $0.45 \mathrm{~g} / \mathrm{L}$ (IQR 0.29-0.73) which was lower than that in the Cardiovascular Health Study $(0.47 \mathrm{~g} / \mathrm{L} ; \mathrm{IQR} 0.41-0.54)$. Furthermore, if we only selected participants older than 65 years in our study, we found that the median fetuin A level was even lower (0.36 g/L; IQR 0.24-0.67). On the other hand, the fetuin A level has different clinical implications in patients undergoing 
dialysis and the general population. For example, dialysis patients with a lower fetuin A level have been reported to suffer from higher $\mathrm{CV}$ mortality, $[11,20,35]$ and in the general population a lower fetuin A level has also been reported to lead to higher CV calcification.[36] However, in patients with diabetes, those with a higher fetuin A level have been reported to experience more $\mathrm{CV}$ complications. [37, 38] It is premature to recognize this phenomenon as another "reverse epidemiology"; nevertheless, the different impact of fetuin A level on incident fractures in different populations can rationally be anticipated. Second, our participants were much younger ( $59 \pm 13$ years) than those in the Cardiovascular Health Study $(74.9 \pm 5.3$ years). According to our results, the risk of major fractures increased by $20 \sim 30 \%$ as the dialysis patients became older (every 10-year increase) (Table 3). In addition, a classical, large cohort study reported that patients in the general population older than 75 years had a higher odds of incident osteoporotic fractures.[39] Although it was underpowered to performed the subgroup analysis in this cohort because only 216 patients were older than 65 years old, we also found a trend of declining impacts of fetuin A levels on the incidence of major fracture in patients older than 65 year old (data not shown). And as we mentioned above, elderly CKD subjects have every likelihood that having osteoporotic fracture. Therefore, the impact of fetuin A level on fractures may be altered by the aging process.

Since fetuin A inhibits the VC process, [11, 40, 41] fetuin A and VC may be potential factors involved in the common pathway of the pathogenesis of fragile fractures in dialysis patients. However, little is known about the physiological link between fetuin A level and the occurrence of fractures. In vitro experiments, bone re-mineralization cannot successfully be performed in fetuin A-depleted serum, although it can be achieved when the serum is reconstituted with fetuin A. $[12,13]$ This suggests that fetuin A promotes bone mineralization in vitro. However, in fetuin A knock-out mice, the trabecular bone mass of cortical bone are unaffected by the absence of fetuin A; nevertheless, there is excess mineralization of the growth plate of long bone[17]. These conflict results from bench studies suggest that the pathophysiology between of fetuin $\mathrm{A}$ and bone mineralization remains unclear and it needs further works to be elucidated. In the view of epidemiological aspects, dialysis patients with evident VC had low bone volume[10] which led to the fragile fracture process, and concurrently, they have lower fetuin A levels as well due to a consumption process[42, 43], as also seen in our results (Table 2). We hypothesize that dialysis subjects with evident VC or having low fetuin A concentration would have more incident fracture owing to either their essential low bone volume or the lack of fetuin A to promote bone mineralization. In addition, sclerostin, a Wnt signaling pathway inhibitor, has been shown to be an important messenger in the cross-talk between bone and the vasculature, and the VC and fractures as well. $[44,45]$ In one phase II study, using romosozumab (a humanized monoclonal antibody to sclerostin) to treat 419 osteoporotic women, the results are promising; however the long-term ( $>12$ months) effect is unknown.[46] Furthermore, a sclerostin inhibitor probably worsens VC in CKD rats [47] and may lead to deterioration of human renal function [33]. However, the association between fetuin A and sclerostin (or Wnt pathway) remains unknown. Therefore, further studies to investigate the potential biological pathway between fetuin A deficiency and the occurrence of incident fractures are warranted.

The strengths of this study are its prospective nature, balanced distribution of gender and available measurements of important confounding variables for outcome analysis, especially GNRI and hs-CRP levels and consistent results while performing both general Cox regression and competing risk models. However, there are some limitations to this study. First, the observational nature of this study precludes the conclusions of a causal relationship. Second, we did not check the BMD of the participants. Although the ability of BMD, as measured by dualenergy X-ray absorptiometry, in dialysis patients to predict the risk of fractures is weak,[31, 48] 
however, a recent study has confirmed the correlation between fetuin A level and BMD in the elderly. We could not fully confirm the correlation between fetuin A, BMD and fractures in dialysis patients from our results due to the lack of BMD measurements. Third, we only identified the presence of aortic arch calcification at entry and did not further quantify the severity of calcification. Dialysis patients with different severities of VC have been reported to have different fetuin A levels, $[38,42]$ and thereby potentially different risks of fractures. It would be more precise to quantify VC to better understand the interrelationship between fetuin A level, VC and fractures. Fourth, this was a single-center study, and all of the participants were treated by the same physicians and underwent uniform laboratory measurements during the observation period, which guaranteed the accuracy of our results. However, our conclusions cannot be generalized to other ethnicities. In summary, our results suggest that lower fetuin A level and the presence of aortic arch calcification can independently predict the long-term occurrence of incident fractures in prevalent dialysis patients.

\section{Supporting Information}

S1 Fig. Distribution of fetuin A concentrations in dialysis patients in the study $(n=685)$. A scatter plot of log hazard ratio (HR) of major incident fracture versus fetuin A concentration with Lowess smoothed function. The plot suggested nonlinear relationships; we therefore categorized patients into tertiles by fetuin A concentration for analyses.

\section{Author Contributions}

Conceived and designed the experiments: HYC. Performed the experiments: HYC YLC YSP. Analyzed the data: HYC JYY. Contributed reagents/materials/analysis tools: HYC YLC SPH MFP JYY. Wrote the paper: HYC.

\section{References}

1. Ball AM, Gillen DL, Sherrard D, Weiss NS, Emerson SS, Seliger SL, et al. Risk of hip fracture among dialysis and renal transplant recipients. JAMA: the journal of the American Medical Association. 2002; 288(23):3014-8. PMID: 12479766.

2. Jadoul M, Albert JM, Akiba T, Akizawa T, Arab L, Bragg-Gresham JL, et al. Incidence and risk factors for hip or other bone fractures among hemodialysis patients in the Dialysis Outcomes and Practice Patterns Study. Kidney international. 2006; 70(7):1358-66. doi: 10.1038/sj.ki.5001754 PMID: 16929251.

3. Beaubrun AC, Kilpatrick RD, Freburger JK, Bradbury BD, Wang L, Brookhart MA. Temporal trends in fracture rates and postdischarge outcomes among hemodialysis patients. Journal of the American Society of Nephrology: JASN. 2013; 24(9):1461-9. doi: 10.1681/ASN.2012090916 PMID: 23744885; PubMed Central PMCID: PMC3752946.

4. Braithwaite RS, Col NF, Wong JB. Estimating hip fracture morbidity, mortality and costs. Journal of the American Geriatrics Society. 2003; 51(3):364-70. PMID: 12588580.

5. Tentori F, McCullough K, Kilpatrick RD, Bradbury BD, Robinson BM, Kerr PG, et al. High rates of death and hospitalization follow bone fracture among hemodialysis patients. Kidney international. 2014; 85 (1):166-73. doi: 10.1038/ki.2013.279 PMID: 23903367; PubMed Central PMCID: PMC3910091.

6. Danese MD, Kim J, Doan QV, Dylan M, Griffiths R, Chertow GM. PTH and the risks for hip, vertebral, and pelvic fractures among patients on dialysis. American journal of kidney diseases: the official journal of the National Kidney Foundation. 2006; 47(1):149-56. doi: 10.1053/j.ajkd.2005.09.024 PMID: 16377396.

7. Rossier A, Pruijm M, Hannane D, Burnier M, Teta D. Incidence, complications and risk factors for severe falls in patients on maintenance haemodialysis. Nephrology, dialysis, transplantation: official publication of the European Dialysis and Transplant Association-European Renal Association. 2012; 27(1):352-7. doi: 10.1093/ndt/gfr326 PMID: 21652549. 
8. London GM, Marty C, Marchais SJ, Guerin AP, Metivier F, de Vernejoul MC. Arterial calcifications and bone histomorphometry in end-stage renal disease. Journal of the American Society of Nephrology: JASN. 2004; 15(7):1943-51. PMID: 15213285.

9. Tomiyama $\mathrm{C}$, Carvalho AB, Higa A, Jorgetti V, Draibe SA, Canziani ME. Coronary calcification is associated with lower bone formation rate in CKD patients not yet in dialysis treatment. Journal of bone and mineral research: the official journal of the American Society for Bone and Mineral Research. 2010; 25 (3):499-504. doi: 10.1359/jbmr.090735 PMID: 19594321.

10. Adragao T, Herberth J, Monier-Faugere MC, Branscum AJ, Ferreira A, Frazao JM, et al. Low bone volume-a risk factor for coronary calcifications in hemodialysis patients. Clinical journal of the American Society of Nephrology: CJASN. 2009; 4(2):450-5. doi: 10.2215/CJN.01870408 PMID: 19158372; PubMed Central PMCID: PMC2637600.

11. Ketteler $M$, Bongartz $P$, Westenfeld $R$, Wildberger JE, Mahnken $A H$, Bohm R, et al. Association of low fetuin-A (AHSG) concentrations in serum with cardiovascular mortality in patients on dialysis: a crosssectional study. Lancet. 2003; 361(9360):827-33. doi: 10.1016/S0140-6736(03)12710-9 PMID: 12642050.

12. Price $P A$, June $H H$, Hamlin NJ, Williamson MK. Evidence for a serum factor that initiates the re-calcification of demineralized bone. The Journal of biological chemistry. 2004; 279(18):19169-80. doi: 10. 1074/jbc.M307880200 PMID: 14978037.

13. Toroian $D$, Price $P A$. The essential role of fetuin in the serum-induced calcification of collagen. Calcified tissue international. 2008; 82(2):116-26. doi: 10.1007/s00223-007-9085-2 PMID: 18097630.

14. Jahnen-Dechent $W$, Heiss $A$, Schafer $C$, Ketteler M. Fetuin-A regulation of calcified matrix metabolism. Circ Res. 2011; 108(12):1494-509. doi: 10.1161/CIRCRESAHA.110.234260 PMID: 21659653.

15. Jahnen-Dechent W, Schafer C, Ketteler M, McKee MD. Mineral chaperones: a role for fetuin-A and osteopontin in the inhibition and regression of pathologic calcification. J Mol Med (Berl). 2008; 86 (4):379-89. doi: 10.1007/s00109-007-0294-y PMID: 18080808.

16. Kapustin AN, Chatrou ML, Drozdov I, Zheng Y, Davidson SM, Soong D, et al. Vascular smooth muscle cell calcification is mediated by regulated exosome secretion. Circ Res. 2015; 116(8):1312-23. doi: 10. 1161/CIRCRESAHA.116.305012 PMID: 25711438.

17. Seto J, Busse B, Gupta HS, Schafer C, Krauss S, Dunlop JW, et al. Accelerated growth plate mineralization and foreshortened proximal limb bones in fetuin-A knockout mice. PLoS One. 2012; 7(10): e47338. doi: 10.1371/journal.pone.0047338 PMID: 23091616; PubMed Central PMCID: PMCPMC3473050.

18. Ix JH, Wassel CL, Bauer DC, Toroian D, Tylavsky FA, Cauley JA, et al. Fetuin-A and BMD in older persons: the Health Aging and Body Composition (Health $A B C$ ) study. Journal of bone and mineral research: the official journal of the American Society for Bone and Mineral Research. 2009; 24(3):51421. doi: 10.1359/jbmr.081017 PMID: 19016589; PubMed Central PMCID: PMC2659522.

19. Fink HA, Buzkova P, Garimella PS, Mukamal KJ, Cauley JA, Kizer JR, et al. Association of Fetuin-A with Incident Fractures in Community-Dwelling Older Adults: The Cardiovascular Health Study. Journal of bone and mineral research: the official journal of the American Society for Bone and Mineral Research. 2015. doi: 10.1002/jbmr.2475 PMID: 25656814.

20. Chen HY, Chiu YL, Chuang YF, Hsu SP, Pai MF, Lai CF, et al. Association of low serum fetuin A levels with poor arteriovenous access patency in patients undergoing maintenance hemodialysis. Am J Kidney Dis. 2010; 56(4):720-7. Epub 2010/08/31. S0272-6386(10)01083-8 [pii] doi: 10.1053/j.ajkd.2010. 06.015 PMID: 20801568.

21. Chen HY, Chiu YL, Hsu SP, Pai MF, Lai CF, Peng YS, et al. Association of serum fetuin A with truncal obesity and dyslipidemia in non-diabetic hemodialysis patients. Eur J Endocrinol. 2009; 160(5):77783. doi: 10.1530/EJE-08-0813 PMID: 19228823.

22. Chen HY, Tsai WC, Chiu YL, Hsu SP, Pai MF, Yang JY, et al. Triglyceride to high-density lipoprotein cholesterol ratio predicts cardiovascular outcomes in prevalent dialysis patients. Medicine (Baltimore). 2015; 94(10):e619. doi: 10.1097/MD.0000000000000619 PMID: 25761189; PubMed Central PMCID: PMCPMC4602469.

23. Chiu YL, Chen HY, Chuang YF, Hsu SP, Lai CF, Pai MF, et al. Association of uraemic pruritus with inflammation and hepatitis infection in haemodialysis patients. Nephrol Dial Transplant. 2008; 23 (11):3685-9. doi: 10.1093/ndt/gfn303 PMID: 18515654.

24. Yamada K, Furuya R, Takita T, Maruyama Y, Yamaguchi Y, Ohkawa S, et al. Simplified nutritional screening tools for patients on maintenance hemodialysis. Am J Clin Nutr. 2008; 87(1):106-13. PMID: 18175743.

25. Gordon L, Olshen RA. Tree-structured survival analysis. Cancer Treat Rep. 1985; 69(10):1065-9. PMID: 4042086. 
26. Fine JP, Gray RJ. A Proportional Hazards Model for the Subdistribution of a Competing Risk. Journal of the American Statistical Association. 1999; 94(446):496-509. doi: 10.1080/01621459.1999.10474144

27. Carballido-Gamio J, Harnish R, Saeed I, Streeper T, Sigurdsson S, Amin S, et al. Structural patterns of the proximal femur in relation to age and hip fracture risk in women. Bone. 2013; 57(1):290-9. doi: 10. 1016/j.bone.2013.08.017 PMID: 23981658; PubMed Central PMCID: PMC3809121.

28. Rabier B, Heraud A, Grand-Lenoir C, Winzenrieth R, Hans D. A multicentre, retrospective case-control study assessing the role of trabecular bone score (TBS) in menopausal Caucasian women with low areal bone mineral density (BMDa): Analysing the odds of vertebral fracture. Bone. 2010; 46(1):17681. doi: 10.1016/j.bone.2009.06.032 PMID: 19747992.

29. Rudang R, Darelid A, Nilsson M, Mellstrom D, Ohlsson C, Lorentzon M. X-ray-verified fractures are associated with finite element analysis-derived bone strength and trabecular microstructure in young adult men. Journal of bone and mineral research: the official journal of the American Society for Bone and Mineral Research. 2013; 28(11):2305-16. doi: 10.1002/jbmr.1974 PMID: 23658040.

30. Jamal SA, Gilbert J, Gordon C, Bauer DC. Cortical PQCT measures are associated with fractures in dialysis patients. Journal of bone and mineral research: the official journal of the American Society for Bone and Mineral Research. 2006; 21(4):543-8. doi: 10.1359/jbmr.060105 PMID: 16598374.

31. Jamal SA, Hayden JA, Beyene J. Low bone mineral density and fractures in long-term hemodialysis patients: a meta-analysis. American journal of kidney diseases: the official journal of the National Kidney Foundation. 2007; 49(5):674-81. doi: 10.1053/j.ajkd.2007.02.264 PMID: 17472850.

32. Toussaint ND, Elder GJ, Kerr PG. Bisphosphonates in chronic kidney disease; balancing potential benefits and adverse effects on bone and soft tissue. Clin J Am Soc Nephrol. 2009; 4(1):221-33. doi: 10. 2215/CJN.02550508 PMID: 18987295.

33. Ott SM. Therapy for patients with CKD and low bone mineral density. Nat Rev Nephrol. 2013; 9 (11):681-92. doi: 10.1038/nrneph.2013.182 PMID: 24100401.

34. Jamal SA, Bauer DC, Ensrud KE, Cauley JA, Hochberg M, Ishani A, et al. Alendronate treatment in women with normal to severely impaired renal function: an analysis of the fracture intervention trial. $J$ Bone Miner Res. 2007; 22(4):503-8. doi: 10.1359/jbmr.070112 PMID: 17243862.

35. Hermans MM, Brandenburg V, Ketteler M, Kooman JP, van der Sande FM, Boeschoten EW, et al Association of serum fetuin-A levels with mortality in dialysis patients. Kidney international. 2007; 72 (2):202-7. doi: 10.1038/sj.ki.5002178 PMID: 17342178

36. Ix JH, Barrett-Connor E, Wassel CL, Cummins K, Bergstrom J, Daniels LB, et al. The associations of fetuin-A with subclinical cardiovascular disease in community-dwelling persons: the Rancho Bernardo Study. Journal of the American College of Cardiology. 2011; 58(23):2372-9. doi: 10.1016/j.jacc.2011. 08.035 PMID: 22115642; PubMed Central PMCID: PMC3224791.

37. Ix JH, Shlipak MG, Brandenburg VM, Ali S, Ketteler M, Whooley MA. Association between human fetuin-A and the metabolic syndrome: data from the Heart and Soul Study. Circulation. 2006; 113 (14):1760-7. Epub 2006/03/29. CIRCULATIONAHA.105.588723 [pii] doi: 10.1161/ CIRCULATIONAHA.105.588723 PMID: 16567568; PubMed Central PMCID: PMC2776669.

38. Laughlin GA, Cummins KM, Wassel CL, Daniels LB, Ix JH. The association of fetuin-A with cardiovascular disease mortality in older community-dwelling adults: the Rancho Bernardo study. Journal of the American College of Cardiology. 2012; 59(19):1688-96. doi: 10.1016/j.jacc.2012.01.038 PMID: 22554599; PubMed Central PMCID: PMC3345127.

39. van Meurs JB, Dhonukshe-Rutten RA, Pluijm SM, van der Klift M, de Jonge R, Lindemans J, et al. Homocysteine levels and the risk of osteoporotic fracture. The New England journal of medicine. 2004; 350(20):2033-41. doi: 10.1056/NEJMoa032546 PMID: 15141041.

40. Schafer C, Heiss A, Schwarz A, Westenfeld R, Ketteler M, Floege J, et al. The serum protein alpha 2Heremans-Schmid glycoprotein/fetuin-A is a systemically acting inhibitor of ectopic calcification. $\mathrm{J}$ Clin Invest. 2003; 112(3):357-66. doi: 10.1172/JCl17202 PMID: 12897203; PubMed Central PMCID: PMC166290.

41. Scialla JJ, Kao WH, Crainiceanu C, Sozio SM, Oberai PC, Shafi T, et al. Biomarkers of vascular calcification and mortality in patients with ESRD. Clin J Am Soc Nephrol. 2014; 9(4):745-55. doi: 10.2215/ CJN.05450513 PMID: 24458076; PubMed Central PMCID: PMC3974354.

42. Moe SM, Reslerova M, Ketteler M, O'Neill K, Duan D, Koczman J, et al. Role of calcification inhibitors in the pathogenesis of vascular calcification in chronic kidney disease (CKD). Kidney international. 2005; 67(6):2295-304. doi: 10.1111/j.1523-1755.2005.00333.x PMID: 15882271.

43. Wang AY, Woo J, Lam CW, Wang M, Chan IH, Gao P, et al. Associations of serum fetuin-A with malnutrition, inflammation, atherosclerosis and valvular calcification syndrome and outcome in peritoneal dialysis patients. Nephrol Dial Transplant. 2005; 20(8):1676-85. Epub 2005/05/19. gfh891 [pii] doi: 10. 1093/ndt/gfh891 PMID: 15899935. 
44. Drueke TB, Lafage-Proust MH. Sclerostin: just one more player in renal bone disease? Clinical journal of the American Society of Nephrology: CJASN. 2011; 6(4):700-3. doi: 10.2215/CJN.01370211 PMID: 21441122.

45. Modder UI, Hoey KA, Amin S, McCready LK, Achenbach SJ, Riggs BL, et al. Relation of age, gender, and bone mass to circulating sclerostin levels in women and men. Journal of bone and mineral research: the official journal of the American Society for Bone and Mineral Research. 2011; 26(2):3739. doi: 10.1002/jbmr.217 PMID: 20721932; PubMed Central PMCID: PMC3179347.

46. McClung MR, Grauer A, Boonen S, Bolognese MA, Brown JP, Diez-Perez A, et al. Romosozumab in postmenopausal women with low bone mineral density. N Engl J Med. 2014; 370(5):412-20. doi: 10. 1056/NEJMoa1305224 PMID: 24382002.

47. Roman-Garcia P, Carrillo-Lopez N, Fernandez-Martin JL, Naves-Diaz M, Ruiz-Torres MP, CannataAndia JB. High phosphorus diet induces vascular calcification, a related decrease in bone mass and changes in the aortic gene expression. Bone. 2010; 46(1):121-8. doi: 10.1016/j.bone.2009.09.006 PMID: 19772957.

48. Cejka D, Patsch JM, Weber M, Diarra D, Riegersperger M, Kikic Z, et al. Bone microarchitecture in hemodialysis patients assessed by HR-pQCT. Clinical journal of the American Society of Nephrology: CJASN. 2011; 6(9):2264-71. doi: 10.2215/CJN.09711010 PMID: 21737853; PubMed Central PMCID: PMC3358993. 